\title{
Subnanometer-Scale Measurements of the Interaction of Ultrafast Soft X-Ray Free-Electron-Laser Pulses with Matter
}

\author{
Stefan P. Hau-Riege, ${ }^{1, *}$ Henry N. Chapman, ${ }^{1}$ Jacek Krzywinski, ${ }^{2}$ Ryszard Sobierajski, ${ }^{2}$ Saša Bajt, ${ }^{1}$ Richard A. London, ${ }^{1}$ \\ Magnus Bergh, ${ }^{3}$ Carl Caleman, ${ }^{3}$ Robert Nietubyc, ${ }^{2}$ Libor Juha, ${ }^{4}$ Jaroslav Kuba, Eberhard Spiller, ${ }^{1}$ Sherry Baker, ${ }^{1}$ \\ Richard Bionta, ${ }^{1}$ K. Sokolowski Tinten, ${ }^{6}$ Nikola Stojanovic, ${ }^{6}$ Benjawan Kjornrattanawanich, ${ }^{7}$ Eric Gullikson, ${ }^{8}$ \\ Elke Plönjes, ${ }^{9}$ Sven Toleikis, ${ }^{9}$ and Thomas Tschentscher ${ }^{9}$ \\ ${ }^{1}$ Lawrence Livermore National Laboratory, Livermore, California 94550, USA \\ ${ }^{2}$ Institute of Physics PAS, Al. Lotników 32/46, PL-02-668 Warsaw, Poland \\ ${ }^{3}$ Department of Cell and Molecular Biology, Biomedical Centre, Uppsala University, SE-75124 Uppsala, Sweden \\ ${ }^{4}$ Institute of Physics AS CR, Na Slovance 2, 18221 Prague 8, Czech Republic \\ ${ }^{5}$ Czech Technical University, Zikova 4, 16636 Prague, Czech Republic \\ ${ }^{6}$ Institut für Experimentelle Physik, Universität Duisburg-Essen, 47048 Duisburg, Germany \\ ${ }^{7}$ Universities Space Research Association, NSLS Beamline X24C, Upton, New York 11973, USA \\ ${ }^{8}$ Lawrence Berkeley National Laboratory, Berkeley, California 94720, USA \\ ${ }^{9}$ Deutsches Elektronen-Synchrotron DESY, Notkestraße 85, D-22607 Hamburg, Germany
}

(Received 5 September 2006; published 4 April 2007)

\begin{abstract}
At the recently built FLASH x-ray free-electron laser, we studied the reflectivity of $\mathrm{Si} / \mathrm{C}$ multilayers with fluxes up to $3 \times 10^{14} \mathrm{~W} / \mathrm{cm}^{2}$. Even though the nanostructures were ultimately completely destroyed, we found that they maintained their integrity and reflectance characteristics during the 25 -fs-long pulse, with no evidence for any structural changes over lengths greater than $3 \AA$. This experiment demonstrates that with intense ultrafast pulses, structural damage does not occur during the pulse, giving credence to the concept of diffraction imaging of single macromolecules.
\end{abstract}

DOI: 10.1103/PhysRevLett.98.145502

PACS numbers: $61.80 . \mathrm{Cb}, 78.67 .-\mathrm{n}$

Femtosecond pulses from soft x-ray free-electron lasers (FELs) [1] are ideal for directly probing matter at atomic lengths and timescales. An important component of understanding ultrafast light-matter interactions is concerned with the onset of atomic motion which is impeded by the atomic inertia. The delay of structural changes will enable atomic-resolution flash-imaging [2] to be performed at upcoming x-ray FELs (XFELs) $[3,4]$ with pulses intense enough to record the $\mathrm{x}$-ray scattering from single molecules [5]. We explored this ultrafast high-intensity regime with the FLASH soft x-ray FEL [6] by measuring the reflectance of nanostructured multilayer mirrors using pulses with fluences far in excess of the damage threshold.

Overcoming the damage barrier by applying short pulses has significant technological relevance. For $\mathrm{x}$ rays, multilayer coatings can provide optics of higher numerical aperture (resulting in smaller focal spots) than uncoated mirrors. Extremely small focal spots will be required for the application of XFELs to biomolecular imaging or the creation and observation of extreme conditions in matter, such as exotic excited states of atoms and warm-dense plasmas. To date, the smallest x-ray focal spots of $30 \mathrm{~nm}$ diameter at $19.5 \mathrm{keV}$ photon energy have been achieved with thick multilayer structures, operating in a Laue geometry [7]. It is predicted that these multilayer Laue lenses will produce spot sizes below $5 \mathrm{~nm}$, and hence could be used to focus XFEL pulses to achieve x-ray power densities of $3 \times 10^{22} \mathrm{~W} / \mathrm{cm}^{2}$, assuming anticipated XFEL light output [4]. Since these lenses are only several hundred micrometers in diameter, they will be exposed to high incident power densities where damage will occur, but not before carrying out their function.

At the FLASH FEL facility, soft $\mathrm{x}$-rays are produced from short electron pulses passing through an undulator, by the principle of self-amplification of spontaneous emission [8]. The peak spectral brilliance of the source is $10^{28}$ photons $/\left(\mathrm{s} \mathrm{mm}^{2} \mathrm{mrad}^{2} 0.1 \%\right.$ bandwidth) [6], 7 orders of magnitude higher than current third-generation synchrotron sources. The machine operated with pulses of $25 \mathrm{fs}$ duration and energies up to $21 \mu \mathrm{J}$. The wavelength was centered around $32.5 \mathrm{~nm}$ with a bandwidth of $0.5 \%$. Higher harmonics contributions are small, for example, the second and third harmonics carry an intensity of 0.3 to $0.6 \%$ of the fundamental [9]. The prepulse energy is expected to be less than $10^{-9}-10^{-8}$ times the main FEL pulse energy, if existing at all due to the different particular way a SASE FEL operates. There is a strong shot-to-shot variation in the beam energy which roughly follows a Gamma distribution, and the x-ray beam is expected to be linearly polarized [6]. We focused these pulses to $3 \times 10^{14} \mathrm{~W} / \mathrm{cm}^{2}$ onto our nanostructured samples while probing the irradiated structures at the nanometer length scale.

The x-ray reflectivity of periodic nanometer-scale multilayers is highly sensitive to changes in the atomic positions and the refractive indices, making them an ideal choice to study structural changes induced by ultrashort FEL pulses. The large reflectivity results from the simultaneous interaction of the $\mathrm{x}$-ray field with many layers of precise thick- 
nesses, each less than the $\mathrm{x}$-ray wavelength. This resonant reflection from the periodic structure is easily disrupted by any imperfection of the layers. The characteristics of the structure, such as periodicity or layer intermixing, can be precisely determined from the measurement of the reflectivity as a function of incidence angle. These parameters can be measured to a small fraction of the probe wavelength, as is well known in x-ray crystallography where average atomic positions of minerals or proteins are found to less than $0.01 \AA$. Thus, we can explore ultrafast phenomena at length scales much less than the wavelength, and investigate the conditions to overcome the effects of radiation damage by using ultrafast pulses.

For our measurements we fabricated mirrors consisting of ten alternating layers of silicon (257.8 $\AA$ thick) and carbon $(17.5 \AA)$, by sputter deposition onto a silicon wafer. A transmission electron micrograph of a cross section of one mirror is shown in Fig. 1(a). The thin carbon layers appear bright in between the thicker silicon layers. We purposely designed the multilayer to minimize the amount of x-ray absorbing carbon, to provide the maximum penetration of the $32.5 \mathrm{~nm}$ light through the structure. In this way the FEL pulse interacts with the most periods, which results in the narrowest Bragg reflection peak and provides us with the highest sensitivity to structural changes achievable with these materials and wavelength. We achieved a further narrowing of the peak by performing the measurement with the plane of reflection parallel to the electricfield vector of the linearly polarized FEL pulse ( $p$ polarization).

Our experimental arrangement is illustrated in Fig. 1(b) [10]. The beam was focused onto the $\mathrm{Si} / \mathrm{C}$ multilayer sample to a spot with a full-width-at-half-maximum of $17 \pm 2 \mu \mathrm{m}$ at an angle of incidence of the sample of $45^{\circ}$ at the mirror surface. This diameter was determined by measuring the area of the damaged region as a function of incoming pulse energy [11].

Incident FEL pulses with fluences greater than about $0.3 \mathrm{~J} / \mathrm{cm}^{2}$ destroy the multilayer structure and leave craters in the substrate, as shown in the Nomarski differentialinterference-contrast (NDIC) micrograph in Fig. 1(e). This optical microscope is sensitive to phase differences across the sample, allowing us to visualize the gradient of the crater depth profile. The terraces are individual layer groups that are ablated, as can also be seen in crosssectional TEM micrographs in Fig. 1(c). Near the threshold of damage, layers ablate (A), recrystallize (B), and interdiffuse $(\mathrm{C})$, possibly accompanied by melting.

We measured the energy of the reflected pulse, for various off-normal angles of incidence ranging from $38^{\circ}$ to $52^{\circ}$, as a function of pulse energy. Each single-pulse measurement was performed on a clean, unexposed area of the mirror. The angular dependence of the reflectivity is shown in Fig. 2(a) for incident pulses attenuated to less than $0.1 \mathrm{~J} / \mathrm{cm}^{2}$ where the multilayer remained undamaged.
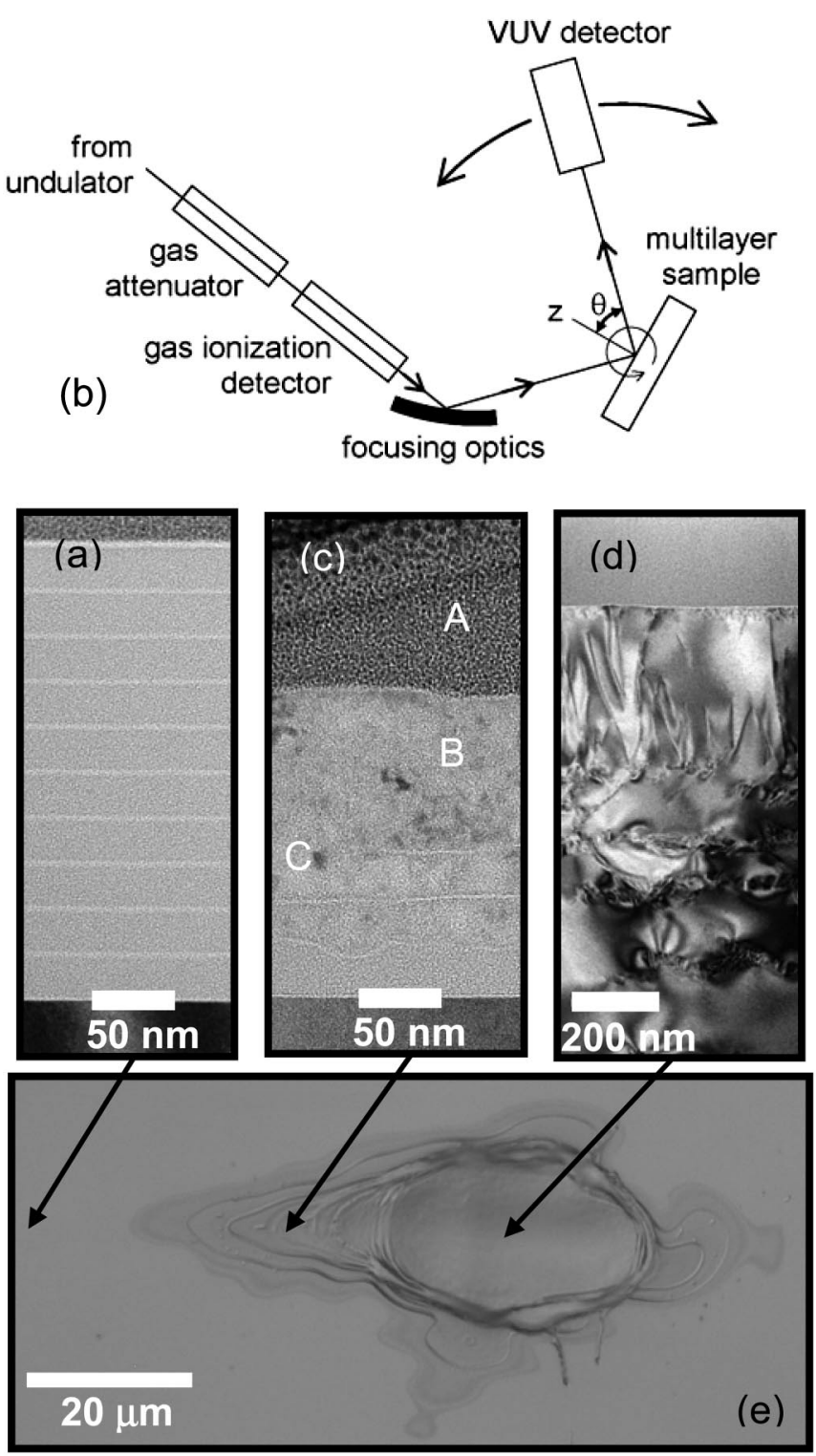

FIG. 1. (a) A TEM image of a cross section of the multilayer nanostructure in an undamaged region, in which the 10 layers of $27.5 \mathrm{~nm}$ period can be seen. (b) Experimental geometry, showing the beam path from FEL undulator through a gas cell (to selectively attenuate the pulse energy by up to a factor of 100) and transparent gas ionization detector (to measure incident pulse energy) before focusing onto the sample with an ellipsoidal mirror. The energy of the pulse reflected from the sample is detected with a photodiode with $1.5^{\circ}$ angular acceptance. (c) and (d) TEM image of cross sections of the nanostructure at different positions within the FEL-exposed spot. The positions are as indicated in (e), which is a NDIC micrograph of the multilayer surface. In (d), the multilayer structure and $400 \mathrm{~nm}$ of the substrate were ablated.

As also shown in Fig. 2(a), the reflectivity is in agreement with that measured at synchrotrons at fluxes substantially below the damage threshold [12] and with calculations. The reflectivity for a larger range of fluences is shown in 

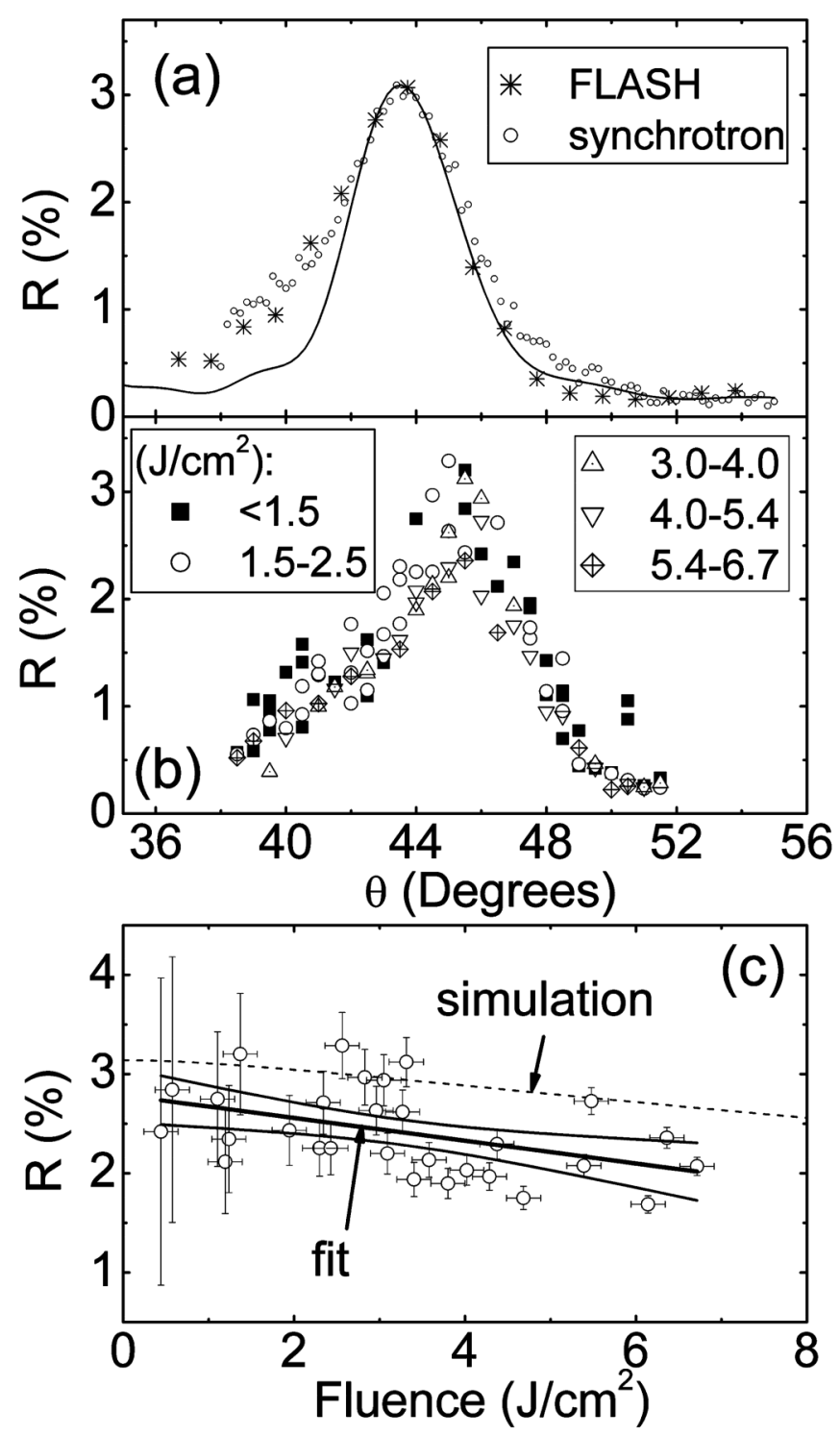

FIG. 2. (a) Low-fluence reflectivity as a function of the offnormal angle of incidence $\theta$ measured at FLASH. Overlaid is the data measured at the synchrotron [11] at a very low flux of less than $1.5 \mu \mathrm{W} / \mathrm{cm}^{2}$ and the calculated reflectivity (solid line). For both we assumed that the incident light consisted of $90 \%$ $p$-polarized light and 10\% s-polarized light. (b) Reflectivity as a function of the off-normal angle of incidence $\theta$ at higher fluences. In this second set of experiments, we observed an angular shift of $1^{\circ}$ with respect to the measurements shown in (a). This could be due to a difference in wavelength of about $0.6 \mathrm{~nm}$, which is consistent wavelength fluctuations at FLASH. The error in the scattering angle is about $1^{\circ}$, and the error in the measured reflectivity varies between less than $0.5 \%$ at the lowest fluence range to less than $0.1 \%$ at the highest fluence range. (c) Multilayer reflectivity ranging from $44^{\circ}$ to $47^{\circ}$ [data taken from (b)] as a function of pulse energy. A linear fit through the data is shown as a thick solid line, and the $90 \%$ confidence level for this fit as thin lines. This fit suggests that the reflectivity drop at the high-fluence end lies between $7 \%$ and $41 \%$. Overlaid as a dashed line are the simulation results.
Fig. 2(b), grouped according to the incident pulse fluence. We found that for fluences up to $6.6 \mathrm{~J} / \mathrm{cm}^{2}$, the angular position of the peak reflectivity does not change by more than the measurement accuracy of $1^{\circ}$, corresponding to a change in period of no more than $3 \AA$. Figure 2(c) shows the reflectivity within the range $44^{\circ} \leq \theta \leq 47^{\circ}$ plotted as a function of fluence. The reflectivity of the multilayer decreases somewhat with increasing fluence.

We have gained a deeper understanding of the multilayer damage mechanism through numerical simulations using hot dense plasma models. Since the sample enters the regime of warm-dense matter (WDM), which is generally not very well understood, we needed to extend physical models originally developed for hot dense plasmas into the WDM regime. The standing-wave intensity distribution inside the multilayer structure was calculated using steady-state methods based on Fresnel equations [13]; these time-independent models are applicable since the time for the light to propagate a few attenuation lengths (approximately $2 \mathrm{fs}$ ) is much shorter than the pulse length ( $25 \mathrm{fs}$ ). We based our estimate of the complex index of refraction of the multilayer materials on published roomtemperature solid-density values [14]. To model the variation with temperature and density, we applied multiplicative correction factors to the opacities based on the XSN opacity model - an average-ion screened-hydrogen model [15]. The correction factors were calculated from the ratio of the XSN opacity at a given temperature and density relative to the XSN opacity at room-temperature and solid density. The index of refraction was calculated from the opacities using the Kramers-Kronig dispersion formula. The soft x-ray beam is absorbed primarily by the electrons through bound-free absorption. Only when the electron temperature reaches $10 \mathrm{eV}$ does free-free absorption become larger than bound-free absorption in silicon. In carbon, this crossover occurs at an electron temperature of $35 \mathrm{eV}$ which was not reached in these experiments. Multiphoton absorption is not expected to have a significant effect. Finally, we calculated the hydrodynamic explosion of the multilayer using the HYDRA radiation hydrodynamics code [16]. We use the QEOS equationof-state (EOS) model to find the pressure and energy of the material [17]. For the conditions of our experiment ( $T_{e} \leq 30 \mathrm{eV}, T_{\text {ion }} \ll T_{e}$, and solid density), the electrons dominate the EOS. They are described by a Thomas-Fermi model with an additional correction for chemical binding. Electron transport and electron-ion coupling are described in the relaxation time approximation, where the momentum transfer cross sections are calculated from Coulomb cross sections with cutoff parameters determined from partial-wave calculations. We found that heat conduction and radiation transport have little effect on the explosion dynamics during the pulse.

Our calculations show that the $32.5 \mathrm{~nm}$ radiation light first heats the electrons in the multilayer to temperatures as 


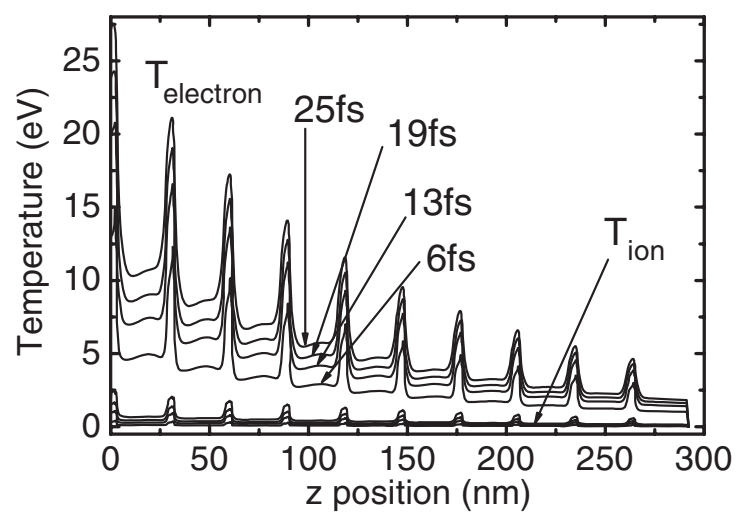

FIG. 3. Computational results for the spatial dependence of electron and ion temperatures at different times during the pulse, assuming a pulse energy of $20 \mu \mathrm{J}$, a pulse length of $25 \mathrm{fs}$, and a beam diameter of $17 \mu \mathrm{m}$.

high as $28 \mathrm{eV}$, as shown in Fig. 3. The nodes of the light intensity reside in strongly absorbing carbon-containing layers, whereas the high-intensity antinodes reside in the more transparent silicon. Since the electrons are not able to transfer a significant amount of energy to the ions before the pulse ends, the ions do not move initially. The multilayer stays intact during the pulse but explodes on a time scale of picoseconds: The pulse-averaged thermal velocity of a carbon atom is $v \approx\left\langle\sqrt{3 k T_{\text {ion }} / m_{\text {ion }}}\right\rangle \sim 0.031 \AA / \mathrm{fs}$; during the $25 \mathrm{fs}$ pulse, the ions can move only about $0.8 \AA$, so that layer interdiffusion is small. The pulseaveraged ion-acoustic velocity of a carbon atom is $v \approx$ $\left\langle\sqrt{3 Z k T_{e} / m_{\text {ion }}}\right\rangle \sim 0.23 \AA /$ fs for an average ionization $Z=$ 2 ; during the 25 fs pulse, the multilayer expands by only about $5.7 \AA$. Since this is much less than the smallest layer thickness it is not expected that the multilayer will be significantly distorted. Therefore, the drop in reflectivity for large fluences as shown in Fig. 2(c) is not likely to be caused by atomic motion. In agreement with our numerical calculation, this reflectivity drop is primarily a consequence of change in the index of refraction induced by ionization and electron heating. Our calculations show the opacity of silicon to increase with temperature due to enhanced free-free photon absorption, leading to less penetration of the light into the multilayer and reduced reflectivity. We calculate the reflectivity to drop by a factor of about 0.5 by the end of the pulse. The pulse-averaged reflectivity drops by only a factor of 0.2 , as indicated in Fig. 2(c). Future modeling and experiments need to focus on obtaining improved opacities in this dense plasma regime in order to better understand the reflectivity drop.

Our experiment shows that precise nanometer-scaled resonantly reflecting structures maintain their integrity and function during irradiation by intense soft x-ray FEL pulses. Structural damage has been observed to occur after the pulse. Important applications of this concept are single molecule diffraction, where uncrystallized macromolecules could be imaged at doses 5 orders of magnitude above the conventional damage threshold [5], and in greatly extending the capabilities of FELs through the use of single-pulse optics.

We are greatly indebted to the machine operators, run coordinators, scientific, and technical teams at the FLASH facility for enabling an outstanding performance. We thank R. W. Lee, M. Marinak, and R. Tipton for helpful discussion, and J. Alameda for fabricating the multilayer structures. This work was performed under the auspices of the U.S. Department of Energy by the University of California, Lawrence Livermore National Laboratory under Contract No. W-7405-Eng-48. This work was partially funded also by the Czech Ministry of Education within the framework of programs INGO (Grant No. 1P2004LA235) and National Research Centers (Projects Nos. LC510 and LC528). The Swedish Research Foundation is acknowledged for financial support.

*To whom all correspondence should be addressed.

[1] J. M. J. Madey, J. Appl. Phys. 42, 1906 (1971).

[2] J. C. Solem and G. C. Baldwin, Science 218, 229 (1982).

[3] M. Altarelli et al., DESY, Hamburg XFEL Technical Design Report No. DESY 2006-067, 2006.

[4] Linac Coherent Light Source (LCLS) SLAC Design Study Report No. SLAC-R-521, 1998, National Technical Information Services, 5285 Port Royal Road, Springfield, Virginia, 22161.

[5] R. Neutze et al., Nature (London) 406, 752 (2000).

[6] V. Ayvazyan et al., Eur. Phys. J. D 37, 297 (2006).

[7] H. C. Kang et al., Phys. Rev. Lett. 96, 127401 (2006).

[8] A. Kondratenko and E. Saldin, Part. Accel. 10, 207 (1980).

[9] S. Duesterer et al., Opt. Lett. 31, 1750 (2006).

[10] K. Tiedtke et al., Proceedings SRI2003, San Francisco, AIP Conf. Proc. No. 705 (AIP, New York, 2004), p. 588.

[11] J. M. Liu, Opt. Lett. 7, 196 (1982).

[12] Advanced Light Source, Beamline 6.3.2. Similar results were obtained at the National Synchrotron Light Source, Beamline X24C.

[13] D. L. Windt, Comput. Phys. 12, 360 (1998).

[14] B. L. Henke, E. M. Gullikson, and J. C. Davis, At. Data Nucl. Data Tables 54, 181 (1993).

[15] R. M. More, J. Quant. Spectrosc. Radiat. Transfer 27, 345 (1982).

[16] M. M. Marinak, Phys. Plasmas 8, 2275 (2001).

[17] R. M. More et al., Phys. Fluids 31, 3059 (1988). 